\title{
EFEITOS DAS INTERVENÇÕES CAMBIAIS A VISTA NA TAXA DE CÂMBIO R\$/US\$: O CASO BRASILEIRO DE 1999 A 2008
}

\author{
Roberto Meurer * \\ Felipe Wolk Teixeira ${ }^{\dagger}$ \\ Eduardo Cardeal Tomazzia $\ddagger$
}

\begin{abstract}
Resumo
Este estudo analisa as intervenções ocorridas no mercado cambial brasileiro entre 1999 e 2008 por meio de operações a vista e seus efeitos na taxa de câmbio R\$/US\$. O período foi segmentado usando os resultados de um modelo MS-VAR. Pelas de estimações GARCH foi possível identificar relação entre intervenções e câmbio, principalmente em subperíodos de menor volatilidade. Estimações com EGARCH indicam assimetria dos choques na volatilidade, os dias de intervenções relacionadas a depreciações apresentam maior efeito na volatilidade que as vinculadas a apreciações. Os resultados indicam a possibilidade de endogeneidade das intervenções. Em muitas situações a força dessas intervenções não foi suficiente para sobrepor a tendência da taxa de câmbio.
\end{abstract}

Palavras-chave: Brasil, intervenções cambiais, volatilidade, taxa de câmbio

\begin{abstract}
This paper analyses effects of interventions in the Brazilian spot foreign exchange market from 1999 to 2008 on the R\$/US\$ exchange rate. The period was divided following the results of a MS-VAR model. The interventions appear to have had an effect on the foreign exchange behavior especially on periods with lower volatility. Estimations with EGARCH point to the existence of asymmetry between interventions: interventions related to positive shocks have a larger effect on the conditional variance than interventions associated to negative shocks. The results indicate the possibility of endogeneity in interventions. Interventions are not able to surpass the trend effects.
\end{abstract}

Palavras-chave: Foreign exchange intervention, volatility, exchange rate, Brazil

JEL classification: E32, E44, F31

\footnotetext{
${ }^{*}$ Departamento de Economia - Universidade Federal de Santa Catarina - UFSCE-mail: rmeurer@mbox1.ufsc.br

† Programa de Pós-Graduação em Economia - Universidade Federal de Santa Catarina - UFSCEmail: felkbr@gmail.com. O autor agradece o apoio da Capes por meio de bolsa de mestrado.

‡ Mestre em Economia pela UFSC e doutorando pelo Programa de Pós-Graduação em Desenvolvimento da Universidade Federal do Paraná - UFSC E-mail: etomazzia@yahoo.com.br
} 


\section{Introdução}

Até que ponto o governo está interessado em interferir na economia por meio da política cambial? O primeiro indício para a resposta é o regime cambial escolhido: câmbio fixo denota completa intervenção, porque o governo define um patamar e age de forma a mantê-lo; o outro extremo é um regime flutuante puro, caracterizado pela ausência de intervenção. O Brasil se encontra entre estes dois extremos, pois adota um regime flutuante sujo desde janeiro de 1999, em que a taxa de câmbio é determinada por forças de mercado, mas ainda assim passa por intervenções do Banco Central, quando este julga oportuno. O argumento principal para as intervenções é o de acomodação de choques internos e externos, uma vez que estes podem ser causadores de flutuações na variável cambial e, consequentemente, nos fundamentos econômicos. Este seria o caso do efeito nos preços dos bens e serviços, uma vez que alta volatilidade cambial gera incerteza para os agentes econômicos, que passam a transferir a volatilidade aos preços, gerando viés inflacionário (Canuto \& Holland 2002).

Um comportamento de turbulência pode ser visto na evolução do mercado de câmbio brasileiro. Na última década, o país passou de um regime de câmbio semi fixo para o flutuante em janeiro de 1999, a moeda brasileira sofreu forte depreciação em 2001, ligada ao contágio da crise argentina e passou por uma mini crise em 2002, devido a incertezas geradas pelo processo eleitoral. Após esse período o câmbio apresentou um longo ciclo de apreciação até entrar em novo processo de depreciação em 2008, resultado da crise financeira mundial.

A possibilidade de suavizar os resultados indesejados dos choques de câmbio, somado ao fato de que essa busca por estabilidade não é a única força motivadora das intervenções, faz com que essas políticas sejam constante objeto de estudos acadêmicos. Torna-se interessante saber até que ponto esse mecanismo afeta o comportamento cambial, por quais canais ele opera e se de fato é eficiente em sua função.

Sarno \& Taylor (2001) mostram a inversão dos resultados empíricos quanto à transmissão de efeitos ao câmbio pelo canal de portfolio (Obstfeld 1990). Até o fim dos anos 1970 e começo dos anos 1980, a literatura demonstrava papel quase nulo, ou então de curtíssimo prazo, para intervenções esterilizadas. Essa posição passa a se alterar a partir dos anos 1990 com os estudos de Dominguez \& Frankel (1993), que demonstram resultados consistentes para essas intervenções. Um dos motivos primários para a inversão, segundo Sarno \& Taylor (2001), foi a evolução na qualidade dos dados disponibilizados.

Porém, ainda não há uma uniformidade de resultados quanto ao efeito das intervenções (Schwartz 2004), o que suscita um questionamento: por que os bancos centrais intervêm se os resultados ainda não são robustos? Pode ser apenas uma falha nos instrumentos de análise utilizados, ou então que os efeitos dessas intervenções sejam inferiores ao conjunto de outros fatores que também agem sobre a flutuação cambial.

Em um estudo mais recente, Dominguez (2006) analisou o efeito de intervenções no câmbio em periodicidade diária e intra-diária nos mercados do Japão, Estados Unidos e Alemanha empregando modelos ARFIMA. O resultado é de que há alteração mais notável na volatilidade cambial no curto prazo de que no longo prazo. Isso incentivaria a realização de intervenções por entidades governamentais, visto que as políticas públicas não visam adicionar 
volatilidade ao câmbio, mas sim suavizá-las (Reinhart 2000).

O presente estudo testa se as intervenções cambiais no mercado brasileiro de câmbio entre 1999 e 2008 afetaram o comportamento da taxa de câmbio R\$/US\$. Para isso, foram estimados modelos de regressão por GARCH e EGARCH para diferentes intervalos de tempo, considerando os ciclos identificados no câmbio. Não é objetivo do trabalho testar a validade da paridade de taxa de juros, mas nas estimativas dos efeitos das intervenções é efetuado o controle para o diferencial entre a taxa de juros interna e externa. Isto significa considerar o argumento de que a taxa de juros incorpora o risco de calote dos títulos da dívida pública (Gonçalves \& Kankzuk 2001), o que implica a relação positiva entre juros domésticos e risco país, já empiricamente comprovada para o Brasil (Guimarães \& Gonçalves 2007, Garcia \& Didier 2003).

Em termos gerais, há relação entre o comportamento cambial e as intervenções no mercado à vista. Também foi identificada assimetria de choques na volatilidade, tal que intervenções relacionadas a depreciações tendem a apresentar maior efeito na volatilidade que as intervenções vinculadas a apreciações. Os resultados indicam a possibilidade de endogeneidade no ato de intervir: as intervenções podem ser condicionadas pelo comportamento cambial e, muitas vezes, a força dessas intervenções não é suficiente para sobrepor esse comportamento.

O trabalho foi organizado em 5 tópicos, incluindo esta introdução. A próxima seção fornece detalhamento sobre as técnicas utilizadas no decorrer da pesquisa. A terceira seção especifica a fonte dos dados e os modelos, na quarta seção são apresentados os resultados das estimações e na quinta são feitos os comentários finais.

\section{Modelo teórico}

Intervenções representam o ato de interferir no mercado de moeda externa, por meio da compra ou venda de moeda externa no mercado spot ou futuro, de forma a alterar o comportamento da taxa de câmbio (Schwartz 2004). As intervenções podem ser divididas em esterilizadas e não-esterilizadas. Estas últimas alteram a base monetária, enquanto as primeiras têm seu efeito sobre a base monetária compensado por intervenção no mercado de moeda (Neely 2005). As intervenções feitas no mercado de câmbio brasileiro são esterilizadas, ou seja, são neutralizadas por mudanças na base monetária, para que a taxa de juros de curtíssimo prazo permaneça nos patamares definidos pela autoridade monetária.

\subsection{O mercado de câmbio brasileiro}

O mercado de câmbio brasileiro é regido pelo Regulamento do Mercado de Câmbio e Capitais Internacionais (RMCCI), instituído pelas Resoluções 3.265/05 e 3.266/05 do Conselho Monetário Nacional e pela Circular no. 3.280/05, do Banco Central do Brasil, que contêm as disposições normativas aplicadas às diversas operações realizadas nesse mercado. De 1988 até 2005, quando foi instituído o RMCCI, o câmbio brasileiro era dividido em dois mercados: o Mercado de Câmbio de Taxas Flutuantes - dólar turismo - e o Mercado de Câmbio de Taxas Livres - dólar comercial -, junto com o segmento de Transferências Internacionais em Reais (TIR). Este último representava os depósitos, em moeda nacional, nas contas de não-residentes no país, as chamadas 
"Contas CC5". A partir de 2005 os dois mercados e o segmento TIR foram unificados, formando um sistema único que engloba todas as operações realizadas. A união permitiu maior transparência nas operações cambiais (Alves \& Simões 2006).

Esse mercado de câmbio unificado é dividido em dois segmentos: o primário e o secundário ou interbancário. No primário são feitas as transações de bens, serviços e ativos entre residentes e não residentes, pelas quais ocorre efetiva entrada ou saída de moeda estrangeira no país (Fernandes 2008). Nesse mercado atuam agentes importadores, exportadores, investidores, agências de turismo, entre outros. As transações são feitas entre esses agentes e um conjunto de instituições financeiras explicitamente autorizadas pelo Banco Central do Brasil (BACEN) a operar no câmbio. Já o mercado interbancário representa um sistema fechado, onde ocorrem as transações apenas entre as diversas instituições autorizadas a operar no câmbio. Todas as operações realizadas nesses mercados são registradas no Sistema de Informações do Banco Central (SISBACEN), de forma a permitir ao BACEN o acompanhamento e fiscalização do mercado. A compra e venda de moeda estrangeira pelo BACEN ocorre no mercado interbancário e é feita pelo Departamento de Operações das Reservas Internacionais (DEPIN), por meio de um conjunto de instituições selecionadas, os dealers .

\subsection{Métodos de estimação}

Nos estudos iniciais de intervenção, como em Humpage (1984), foram estimados modelos lineares similares ao da equação (1), com base em dados diários:

$$
\Delta S_{t}=\beta_{0}+\beta_{1} I_{t}+\beta_{2} x_{1 t}+e_{t},
$$

onde:

- $\Delta S_{t}$ : Variação do câmbio, mensurada como $\Delta S_{t}=\ln \left(S_{t} / S_{t-1}\right)$

- $I_{t}$ : Medida de Intervenção, em volume de moeda;

- $x_{1 t}$ : Conjunto de outras variáveis econômicas que podem explicar a mudança de câmbio.

Nestes modelos, um coeficiente $\beta_{1}$ estatisticamente diferente de zero indica que algum dos movimentos cambiais pode ser explicado pela medida de intervenção. Os resultados dessas estimações em Humpage (1984) não foram significantes. Uma das razões apontadas para isso foi a baixa frequência ou relevância no montante das intervenções, em contraste à grande variação cambial diária.

Uma extensão a esse modelo é dada pela inclusão de uma variável referente ao diferencial de juros doméstico e externo, $i-i *$, como em Dominguez \& Frankel (1993), onde um aumento no diferencial pode causar deslocamento de capital em direção ao mercado doméstico.

É possível também caracterizar as intervenções por meio de dummies. Aplicase uma dummy que assume valor 1 para dias com intervenção e 0 para os demais. Os testes podem ser feitos com a dummy em qualquer data de interesse. A estimação com dummies aplicada ao tema proposto aqui apresenta o seguinte formato geral:

$$
\Delta S_{t}=b_{0}+b_{1} D_{t}
$$


onde $D$ é a dummy para intervenções. O subscrito $t$ indica o período no qual a dummy será inserida, podendo ser no dia da intervenção, períodos anteriores e posteriores.

Outro caminho é testar o efeito da intervenção diretamente na volatilidade do câmbio. Para isso, são utilizados modelos GARCH, de heterocedasticidade condicional auto-regressiva. A hipótese é testar se a intervenção apresenta algum papel na formação da variância condicional da taxa de câmbio. A formulação de um GARCH apresenta um termo de erro $\left(\varepsilon_{t}\right)$ tal que:

$$
\varepsilon_{t}=z_{t} \sqrt{h_{t}}
$$

onde $v_{t}$ é um ruído branco e $h_{t}$ é a variância condicional:

$$
h_{t}=\beta_{0}+\beta_{1} h_{t-1}+\beta_{2} e_{t-1}^{2}+\beta_{3} I_{t}
$$

$I_{t}$ indica a série de intervenções testada.

Uma estimação complementar é o EGARCH (Exponential GARCH), que visa testar a existência de assimetria dos choques na volatilidade, ou seja, se choques negativos e positivos no retorno tem efeitos diferentes na variância condicional. A intuição inicial é buscar associar os choques negativos a intervenções de venda e choques positivos a intervenções de compra: vendas apreciariam a taxa de cambio causando choques negativos no retorno, enquanto compras depreciariam a taxa de câmbio, gerando choques positivos no retorno. A variância condicional no EGARCH é dada por:

$$
\ln \left(h_{t}\right)=\gamma_{0}+\gamma_{1}\left|z_{t-1}\right| \gamma_{2} z_{t-1}+\gamma_{3} \ln \left(h_{t-1}\right)+\gamma_{n} \text { interv, }
$$

onde $z_{t-1}=\varepsilon_{t-1} / \sqrt{h_{t}}$ e $\gamma_{n}$ interv representam as diferentes séries de intervenção testadas na equação de variância.

O impacto dos choques na variância é assimétrico se $\gamma_{2} \neq 0$. Quando $\gamma_{2}>$ 0 , os choques positivos afetam mais intensamente a variância condicional que choques negativos, ou seja, depreciações do real frente ao dólar afetam mais a variância que as apreciações (Araujo 2004).

\section{Dados, modelos utilizados e quebra de períodos do câmbio}

\subsection{Dados}

São três os principais mecanismos de intervenção cambial: leilões no mercado de dólar a vista, negociação de títulos da dívida indexados ao dólar e contratos de swap. O enfoque deste trabalho é nas intervenções no mercado a vista.

O BACEN divulga, no demonstrativo mensal de reservas internacionais, uma série referente às intervenções cambiais no mercado a vista, porém não estão disponíveis dados diários. Utilizou-se, então, a série dos Fatores Condicionantes da Base Monetária - Operações com o Setor Externo, que contabiliza a compra e venda de divisas pelo Banco Central. Essa série não contém apenas as intervenções diretas, incluindo também outras operações, como trânsito de recursos de organismos internacionais destinados à manutenção de suas representações no Brasil. A série dos Fatores Condicionantes da Base Monetária expressa em reais foi convertida em dólares utilizando a taxa de câmbio ptax de venda divulgada pelo Banco Central. A série tem comportamento muito próximo da série de intervenções mensais do Demonstrativo de Reservas Internacionais, com coeficiente de correlação de 0,973. Existe uma defasagem 
de dois dias úteis entre as operações com dólares e a sua liquidação em reais, ou seja, caso o BACEN atue no mercado a vista em um dia D, a conta de Operações com o Setor Externo é sensibilizada em D+2. A série foi ajustada para compensar essa defasagem.

As taxas de juros utilizadas foram a Selic Anualizada, disponibilizada pelo IPEADATA, e a Effective Federal Funds Rate, divulgada pela Board of Governors of the Federal Reserve System (http://research.stlouisfed.org) .

O período de estimação vai de 05/01/1999 até 31/12/2008. Visto que nesse intervalo há modificações econômicas e políticas que afetam o comportamento da taxa de câmbio, a série da taxa de câmbio foi utilizada com a segmentação de acordo com os resultados de um modelo de Mudança de Regimes Markoviano estimado em Meurer et al. (2010), que encontra os subperíodos mostrados na Tabela 1 .

Tabela 1: Subperíodos do câmbio: 1999 a 2008

\begin{tabular}{ll}
1 & $05 / 01 / 1999-25 / 04 / 2002$ \\
2 & $26 / 04 / 2002-11 / 08 / 2003$ \\
3 & $12 / 08 / 2003-08 / 09 / 2008$ \\
4 & $09 / 09 / 2008-31 / 12 / 2008$ \\
\hline
\end{tabular}

O intervalo inicial, de 05/01/1999 a 25/04/2002, vai do início do regime de câmbio flutuante até a crise de confiança gerada pelo aumento das intenções de voto no candidato Luis Inácio Lula da Silva nas pesquisa eleitorais. O intervalo seguinte, 26/04/2002 a 11/08/2003 é caracterizado por alternância de regimes, indicando a volatilidade elevada gerada pela crise de confiança no período eleitoral e o início do governo Lula. A data final desse intervalo é um indicador do período em que ocorre elevação da confiança no governo Lula e convicção na ausência de rupturas na condução da política econômica. O terceiro período, de 12/08/2003 a 08/09/2008, é um ciclo longo de apreciação da moeda brasileira, em que há uma política declarada de aumento das reservas internacionais e redução da exposição cambial do setor público (BACEN, 1998-2007). O último período, de 09/09/2008 a 31/12/2008 é referente a um regime de retornos positivos, vinculado à depreciação do Real frente ao Dólar, durante a crise no sistema financeiro internacional.

\subsection{Modelos utilizados}

O passo inicial antes de serem estimados os modelos foi definir se a variável utilizada deveria ser o câmbio em nível ou a sua primeira diferença. Para isso foram utilizados os testes de raiz unitária Augmented Dickey-Fuller (ADF, Dickey \& Fuller (1979), Dods \& Giles (1995), Elliot, Rothenberg, e Stock ERS, Elliot et al. (1996) e o Ng \& Perron (2001)). De acordo com a tabela 2, para todos os subperíodos, não é rejeitada a hipótese nula de existência de raiz unitária na série em nível. Na série em primeira diferença a hipótese nula é rejeitada. A existência de raiz unitária na série em nível significa a nãoestacionariedade da mesma e a necessidade de uso da primeira diferença da série, representada pelo retorno cambial.

Para cada subperíodo, foram testadas as intervenções de compra, venda e intervenções agregadas (compras e vendas) ; para isso, foram estimadas as seguintes regressões: 
Tabela 2: Testes de Raiz Unitária

\begin{tabular}{|c|c|c|c|c|c|}
\hline \multirow{3}{*}{ Testes } & \multicolumn{5}{|c|}{ Estatísticas dos períodos } \\
\hline & \multirow{2}{*}{ Inteiro } & \multicolumn{4}{|c|}{ Subperíodo } \\
\hline & & 1 & 2 & 3 & 4 \\
\hline ADF (nível) & $-2,944056$ & $-3,773127$ & $-1,858263$ & $-2,958138$ & $-2,637332$ \\
\hline ERS (nível) & 75,14509 & 35,30569 & 77,46311 & 6,989573 & 11,08554 \\
\hline \multicolumn{6}{|l|}{$\begin{array}{l}\text { NG-Perron } \\
\text { (nivel) }\end{array}$} \\
\hline $\mathrm{MZa}$ & $-1,10156$ & $-2,52947$ & $-0,96133$ & $-13,2693$ & $-9,81891$ \\
\hline $\mathrm{MZt}$ & $-0,69047$ & $-1,0393$ & $-0,56272$ & $-2,56233$ & $-2,09739$ \\
\hline MSB & 0,62681 & 0,41088 & 0,58536 & 0,1931 & 0,21361 \\
\hline MPT & 73,5521 & 32,8813 & 67,3793 & 6,94673 & 9,80459 \\
\hline $\begin{array}{l}\text { ADF } \\
\left(1^{\text {a }} \text { diferença }\right)\end{array}$ & $-45,63115^{* * *}$ & $-17,82197^{* * *}$ & $-13,93471^{* * *}$ & $-33,75593^{* * *}$ & $-10,66864^{* * *}$ \\
\hline $\begin{array}{l}\text { ERS } \\
\left(1^{a} \text { diferença }\right)\end{array}$ & $0,073206^{* * *}$ & $0,244134^{* * *}$ & $0,408036^{* * *}$ & $0,213051^{* * *}$ & $2,317429^{* * *}$ \\
\hline \multicolumn{6}{|l|}{$\begin{array}{l}\text { NG-Perron } \\
\text { (1 } 1^{\text {a }} \text { diferença) }\end{array}$} \\
\hline $\mathrm{MZa}$ & $-1244,18^{* * *}$ & $-373,259^{* * *}$ & $-212,301^{* * *}$ & $-19,541^{* * *}$ & $-38,6805^{* * *}$ \\
\hline $\mathrm{MZt}$ & $-24,9417^{* * *}$ & $-13,6605^{* * *}$ & $-10,3029^{* * *}$ & $-3,1204^{* * *}$ & $-4,39743^{* * *}$ \\
\hline MSB & $0,02005^{* * *}$ & $0,0366^{* * *}$ & $0,04853^{* * *}$ & $0,15969^{* * *}$ & $0,11369^{* * *}$ \\
\hline MPT & $0,07324^{* * *}$ & $0,24563^{* * *}$ & $0,42925^{* * *}$ & $4,69703^{* * *}$ & $2,35759^{* * *}$ \\
\hline
\end{tabular}

${ }^{* * *}$ Rejeita a hipótese nula de existência de raiz unitária a 1\%

1. Ret $=b_{0}+b_{1} A R(p)+b_{2}$ vend $a+b_{3}\left(i-i^{*}\right)+e_{t}$

2. $\operatorname{Ret}=b_{0}+b_{1} A R(p)+b_{2} \operatorname{comp}+b_{3}\left(i-i^{*}\right)+e_{t}$

3. $\operatorname{Ret}=b_{0}+b_{1} A R(p)+b_{2} c b n+b_{3}\left(i-i^{*}\right)+e_{t}$

4. Ret $=b_{0}+b_{1} A R(p)+b_{2}$ vend $a+b_{3} \operatorname{comp}+b_{4}\left(i-i^{*}\right)+e_{t}$

Onde:

Ret: Retornos do câmbio;

$A R(p)$ : Componente autoregressivo de grau p;

Venda, comp e cbn: Volume das intervenções de venda de dólares, de compra de dólares e compras e vendas na mesma série, respectivamente;

$\boldsymbol{i}-\boldsymbol{i}^{*}$ : Diferença entre taxa de juros Selic e FedFunds .

A inclusão da diferença entre taxa de juros doméstica e internacional visa captar a influência da condição de paridade internacional de juros sobre os movimentos do câmbio.

Os modelos foram testados por LM-Arch, e em todos foram detectados processos ARCH. Optou-se, mediante os critérios de seleção AIC/SBC, pelo modelo GARCH $(1,1)$, mais parcimonioso. Como o comportamento cambial frequentemente apresenta um comportamento auto-regressivo, foi acrescentado um componente AR de 1 a 5 defasagens mediante avaliação dos critérios de Akaike e Schwarz (AIC e SBC). As variáveis de intervenção também foram testadas com defasagens. Algumas das variáveis não estatisticamente significantes foram removidas com base nos critérios AIC e SBC. 
O modelo 4 é complementar ao 3: em ambos as direções de compra e venda são testadas simultaneamente, porém no modelo 4 as direções são testadas em variáveis separadas. Os modelos 3 e 4 também foram estimados por EGARCH, para testar a existência de assimetria dos choques na volatilidade. Como nos modelos 3 e 4 dos subperíodos foram identificadas assimetrias nos choques, os resultados nas tabelas dos respectivos modelos são referentes à estimação por EGARCH, com exceção do subperíodo 2, devido à não identificação de assimetria.

Os valores das intervenções estão expressos em bilhões de dólares. Foi delimitado um valor mínimo de corte de US\$ 200 milhões. Intervenções com valores inferiores a este valor foram desconsideradas visando reduzir o ruído. No subperíodo 3 não foi possível utilizar o valor de corte nas operações de venda devido a pouca frequência dessas intervenções; uma série com todos os valores, sem cortes, foi utilizada. Os outros períodos também foram estimados com a série sem valor de corte e também com valores intermediários. Os resultados mais robustos foram encontrados com a utilização do corte de US\$ 200 milhões. Isto indica duas possibilidades: (a) a consideração das diversas intervenções com valor de corte baixo pode ser responsável por muito ruído na estimação e/ou (b) : a magnitude da intervenção pode influenciar o comportamento do câmbio.

No subperíodo 3 não foi possível utilizar o valor de corte nas operações de venda devido a pouca frequência dessas intervenções; uma série com todos os valores, sem cortes, foi utilizada. 


\section{Resultados}

\subsection{Resultados das estimativas e análise}

No primeiro subperíodo, de 05/01/1999 a 25/04/2002 (Tabela 3 para equações da média e estatísticas da regressão, Tabela 4 para equações da variância), observa-se que as intervenções de venda estão relacionadas a movimentos na direção oposta do câmbio: uma venda de US\$ 1 bi está vinculada a depreciação cambial de 0,9\% (coeficiente do modelo 1, significante a 1\%) e 0,03\% (modelo 4 , significante a $1 \%$ ). Pode ser visto também aumento na volatilidade relacionado às intervenções nessa direção, conforme os coeficientes das equações de variância dos modelos 1, 3 e 4 (significantes a $10 \%, 5 \%$ e 1\%). Já intervenções de compra de US\$ 1 bilhão estão relacionadas a depreciações de 0,4\% no câmbio dois dias após a intervenção (modelo 2, significante a 5\%) e, segundo o modelo 4 , depreciação de $0,5 \%$ no dia da intervenção (significante a $1 \%)$.

Observa-se que a variável referente ao diferencial de juros interno e externo é significante a $1 \%$ em todos os modelos, e apresenta o sinal esperado: aumentos de $1 \%$ na diferença dos juros causam apreciação cambial de $0,17 \%$ a $0,20 \%$. Segundo os modelos 3 e 4 , choques positivos no câmbio têm maior efeito na variância condicional que choques negativos. Esse impacto é captado pelos coeficientes $\gamma 2$ positivos e significantes a $1 \%$ em ambos os modelos.

Durante o segundo subperíodo, que vai de 26/04/2002 a 11/08/2003 (tabelas 5 para equações da média e estatísticas da regressão e 6 para equações da variância), as intervenções de venda estão relacionadas a movimentos de apreciação cambial. Segundo os modelos 1 e 4, uma venda de US\$ 1 bi está vinculada a apreciação de aproximadamente $0,11 \%$ no câmbio na data de venda (coeficientes significantes a 1\%). As equações da variância mostram indícios fracos de redução de volatilidade associados às datas de operações de venda.

Um resultado interessante é dado pelas intervenções de compra: compras de US\$ 1 bi estão vinculadas a apreciação de $0,13 \%$ (coeficiente significante a $1 \%$ ), segundo o modelo 2; o mesmo coeficiente é significante apenas a $10 \%$ no modelo 4; e o modelo 3 não apresenta coeficientes significantes. A grande instabilidade do período, somada a uma menor frequência e magnitude de intervenções de compra pode ter sido a responsável pelo fraco papel detectado nas estimações, de modo que os súbitos movimentos negativos no retorno, em meio à tendência maior de depreciação, possam ter sido capazes de se sobrepor ao efeito das intervenções de compra de dólares.

O diferencial de juros interno e externo novamente é significante a $1 \%$ em todos os modelos: aumentos de $1 \%$ na diferença dos juros causam apreciação cambial de $0,17 \%$ a $0,20 \%$. Nesse período, não foi possível identificar assimetria nos choques para os modelos 3 e 4 e o modelo inserido nas tabelas é o GARCH. A não captação de assimetria é vinculada à baixa frequência de intervenções de compra.

No terceiro subperíodo, de 12/08/2003 a 08/09/2008 (tabelas 7 e 8, equações da média e variância, respectivamente), não houve relação significante entre intervenções de venda isoladas e o comportamento cambial, tanto para a média como na variância, nos modelos 2 e 4 , que pode ser decorrente da baixa frequência e magnitude das intervenções.

As intervenções de compra apresentam significância tanto na data zero como nas defasagens. É observada uma mudança de sinal nos coeficientes 


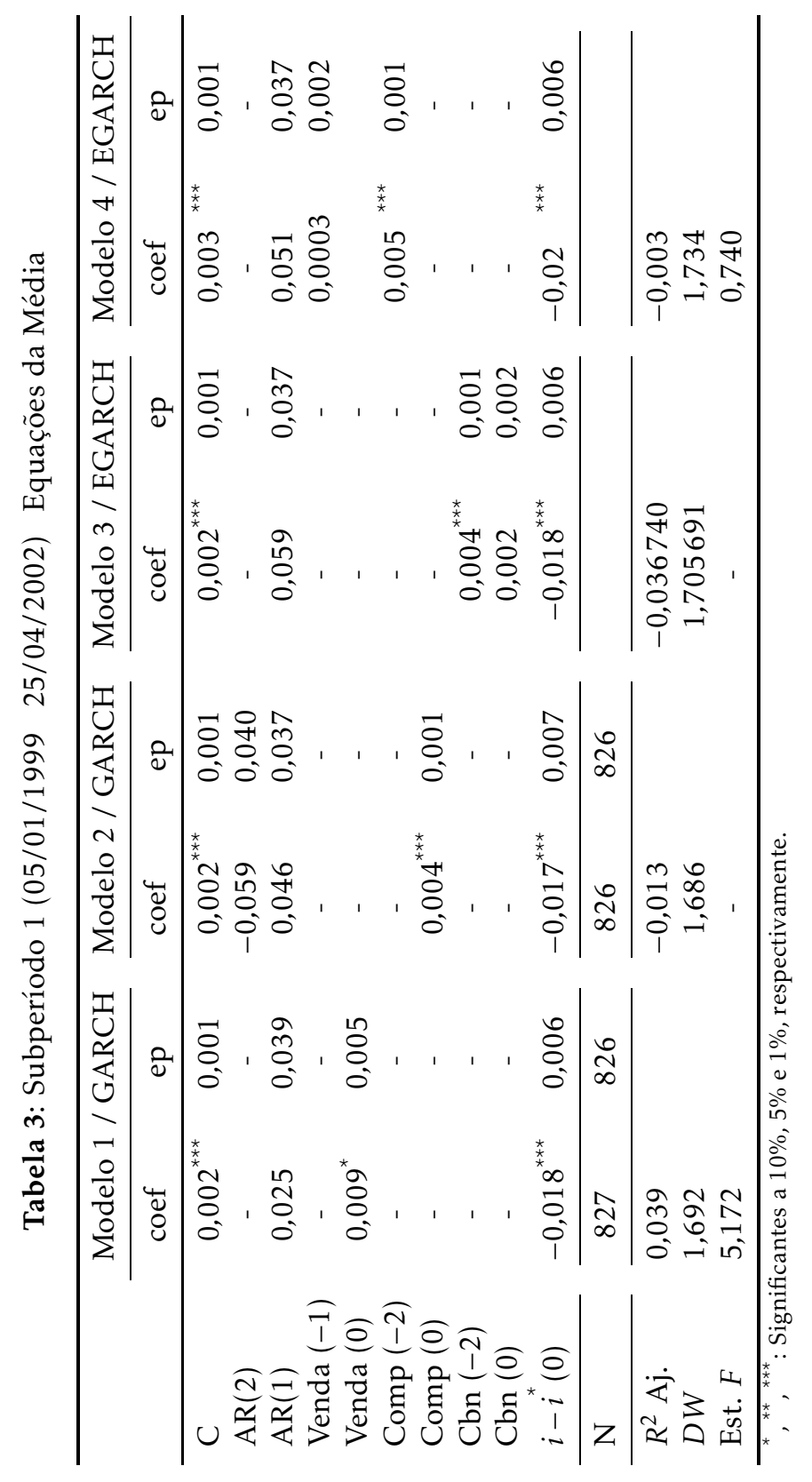




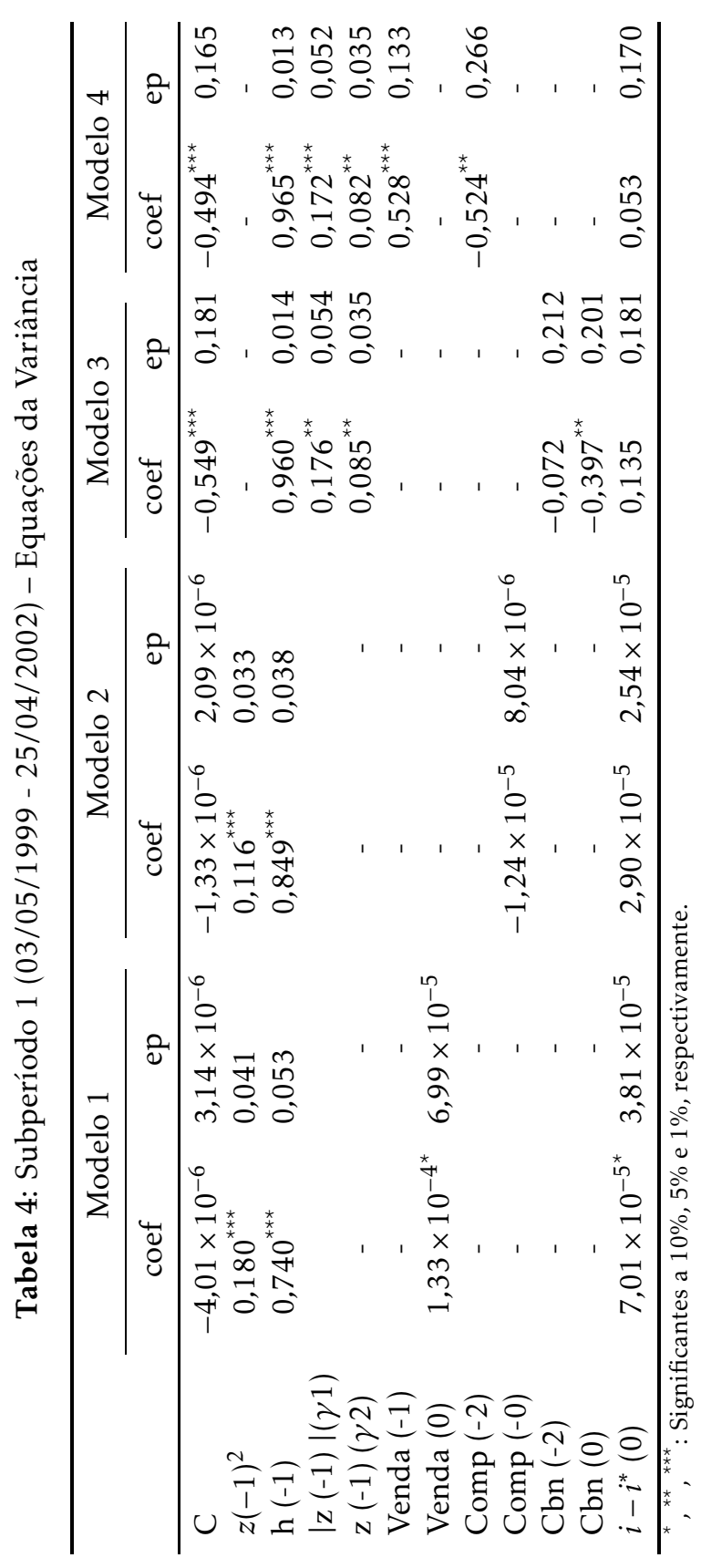




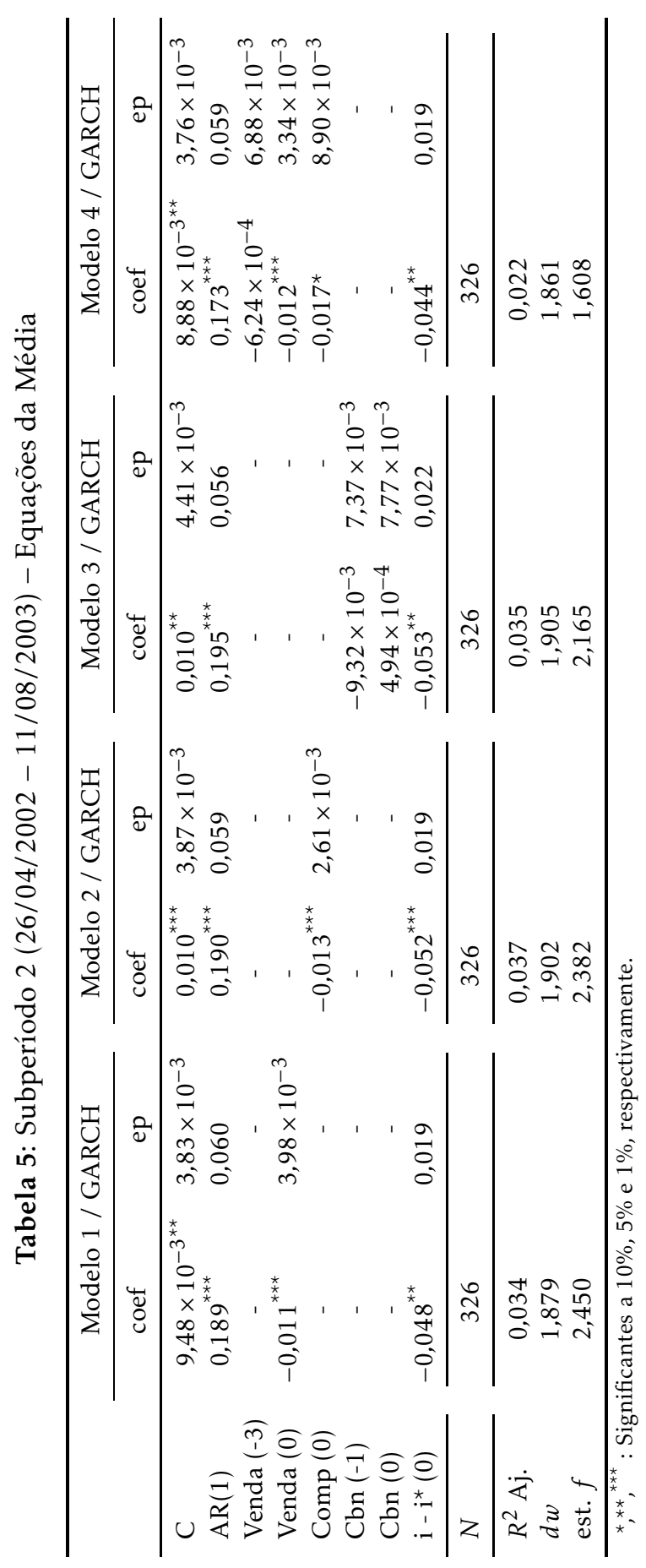




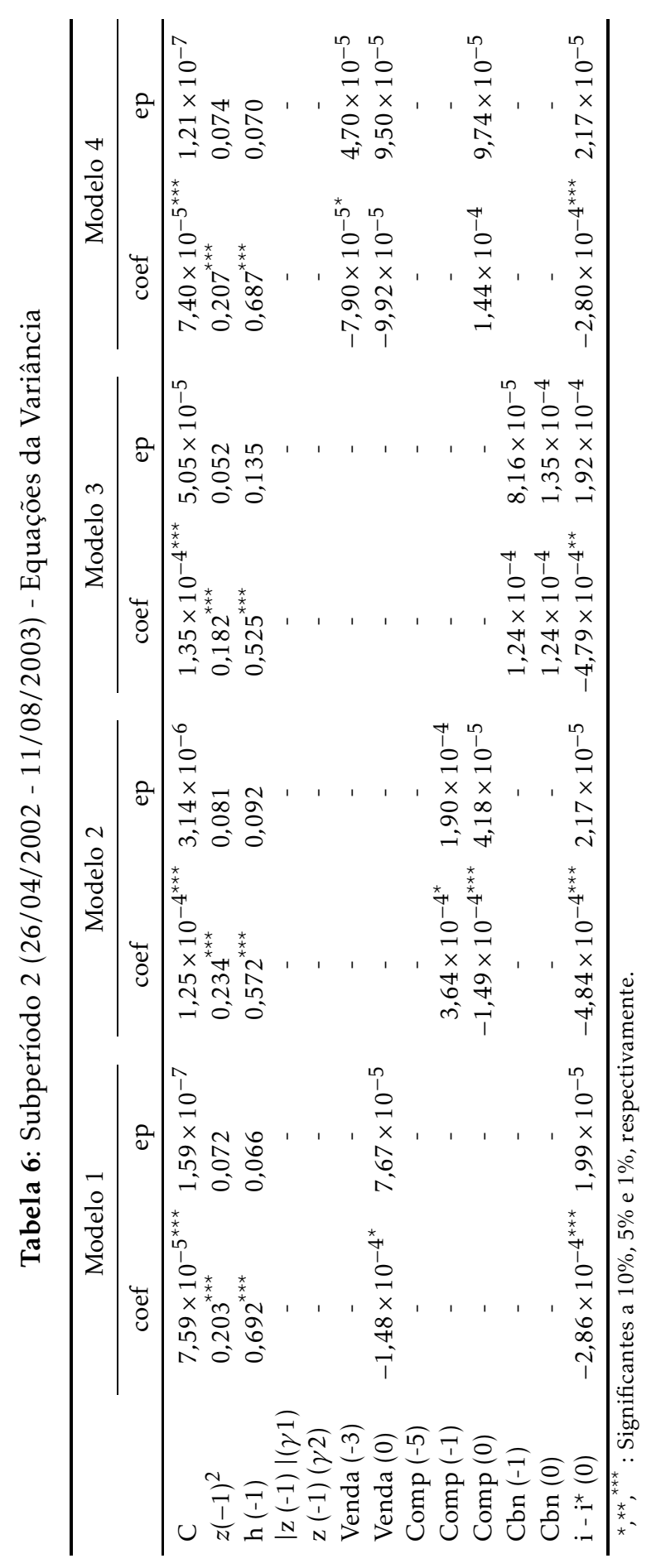


(negativo na data zero e positivo nas defasagens 1 e 2), o que pode representar uma sobre-reação do mercado às intervenções. Uma compra de US\$ 1 bilhão causa, inicialmente (no dia da intervenção) apreciação de $0,55 \%$, sendo acompanhada por depreciação, nos dois dias subsequentes, de 0,31\% e 0,22\%, respectivamente (coeficientes significantes a $1 \%$ ), o que gera um efeito líquido muito próximo de zero. As intervenções nessa direção parecem reduzir a volatilidade cambial segundo os modelos 2 e 4.

O modelo 3 apresenta resultados bastante similares aos obtidos com o modelo 2 com relação à inversão de sinal nos coeficientes. Este resultado e os coeficientes não significantes referentes às intervenções de venda levam à conclusão de que há proeminência do papel das intervenções de compra para esse subperíodo. A variável referente ao diferencial de juros interno e externo é significante a $1 \%$ apenas nesse modelo, um aumento de $1 \%$ no diferencial está vinculado a apreciação cambial de $0,34 \%$. Nesse subperíodo foi detectada assimetria nos choques para os modelos 3 e 4 , choques positivos no câmbio têm maior efeito na variância condicional que choques negativos.

No quarto e último subperíodo, de 09/09/2008 a 31/12/2008 (tabelas 9 e 10 , equações de média e variância, respectivamente), há intensa ação no sentido de intervenções de venda, porém as mesmas não parecem ser capazes de compensar imediatamente o efeito da tendência cambial. Vendas de US\$ 1 bilhão estão relacionadas a depreciação de 1,3 a 1,5\% (modelos 3 e 1, respectivamente, ambos significantes a 1\%) na data da intervenção. No modelo 4 foi possível observar mudança no sinal do coeficiente, indicando uma possível defasagem no efeito dessas intervenções.

Nesse subperíodo, há poucas intervenções de compra, aproximadamente US $\$ 4$ bilhões, com apenas cinco dias com intervenções superiores a US $\$ 200$ milhões. Possivelmente a causa para a divergência entre os resultados obtidos seja que o modelo 2 não identifica relação entre compras e câmbio, enquanto no modelo 4 compras de US\$ 1 bilhão estão relacionadas a depreciação de $3,3 \%$ (significante a 1\%). O modelo 3 possivelmente reflete as operações de venda, que chegaram, no subperíodo, a aproximadamente US\$ 23 bilhões, mostrando, novamente, que as intervenções não são capazes de fazerem frente à tendência cambial. O coeficiente negativo implica que operações de venda estão relacionados a depreciações e operações de compra a apreciações (significante a $1 \%$ ) .

O diferencial de juros interno e externo é significante a $1 \%$ em todos os modelos, onde aumentos nesse diferencial estão relacionados a apreciações. Foi também identificada assimetria nos choques para os modelos 3 e 4, choques positivos no câmbio têm maior efeito na variância condicional que choques negativos.

De forma a complementar aos resultados acima, os modelos também foram estimados para o período integral de 05/01/1999 a 31/12/2008 (tabelas 11 e 12, equações da média e variância, respectivamente). É observado que as intervenções de venda não se mostram capazes de sobrepor a tendência, pois uma venda de US\$ 1 bilhão está associada a depreciação cambial de $0,76 \%$. As intervenções de compra isoladas (modelo 2) apresentam significância tanto na data zero como nas defasagens. Há mudança de sinal nos coeficientes, negativo na data zero e positivo na primeira defasagem. Isto pode significar uma sobre-reação do mercado às intervenções, como já mencionado. Uma compra de US\$ 1 bilhão causa, inicialmente (no dia da intervenção) apreciação de $0,31 \%$, sendo acompanhada por depreciação de aproximadamente $0,12 \%$ 


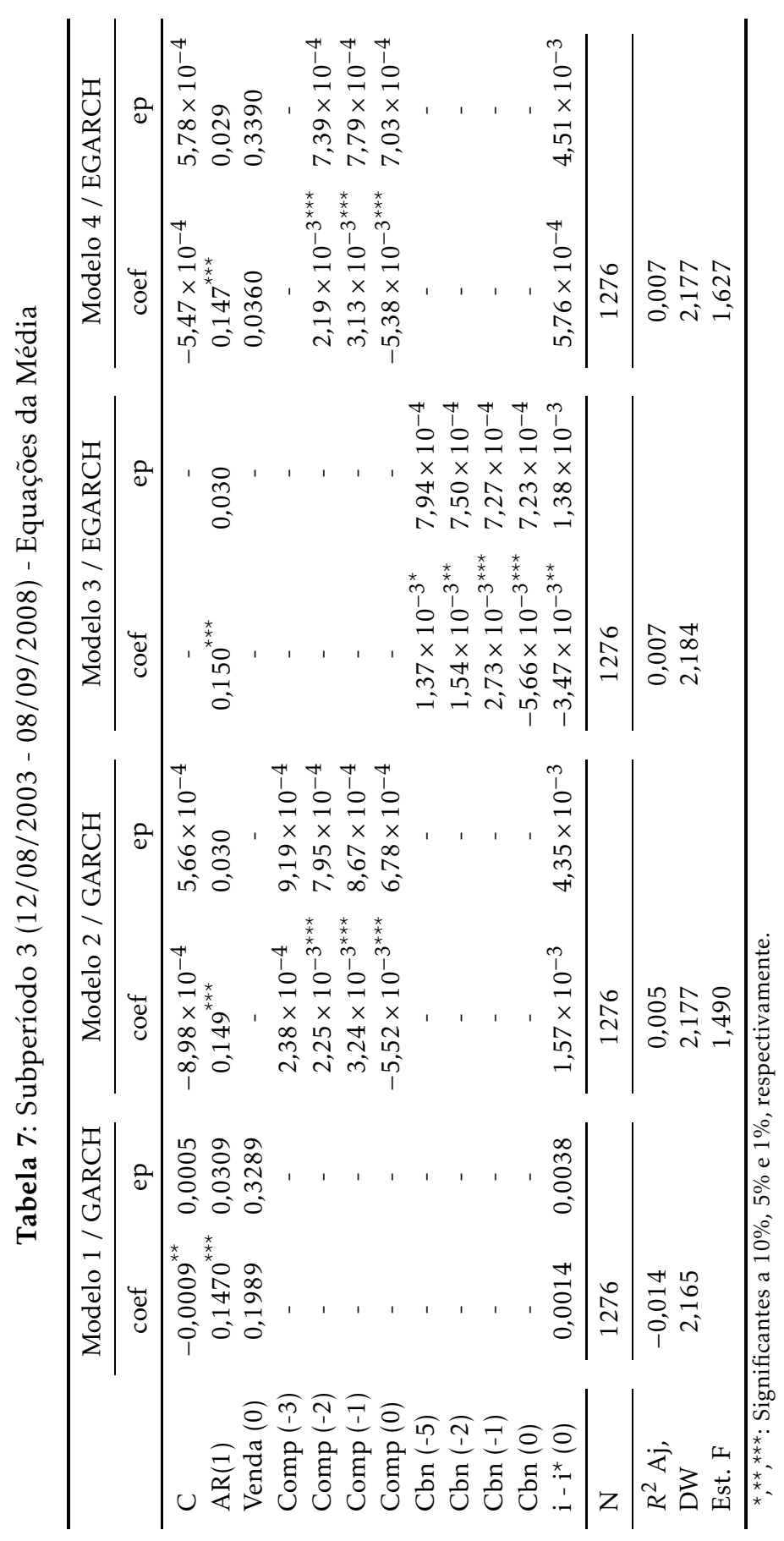




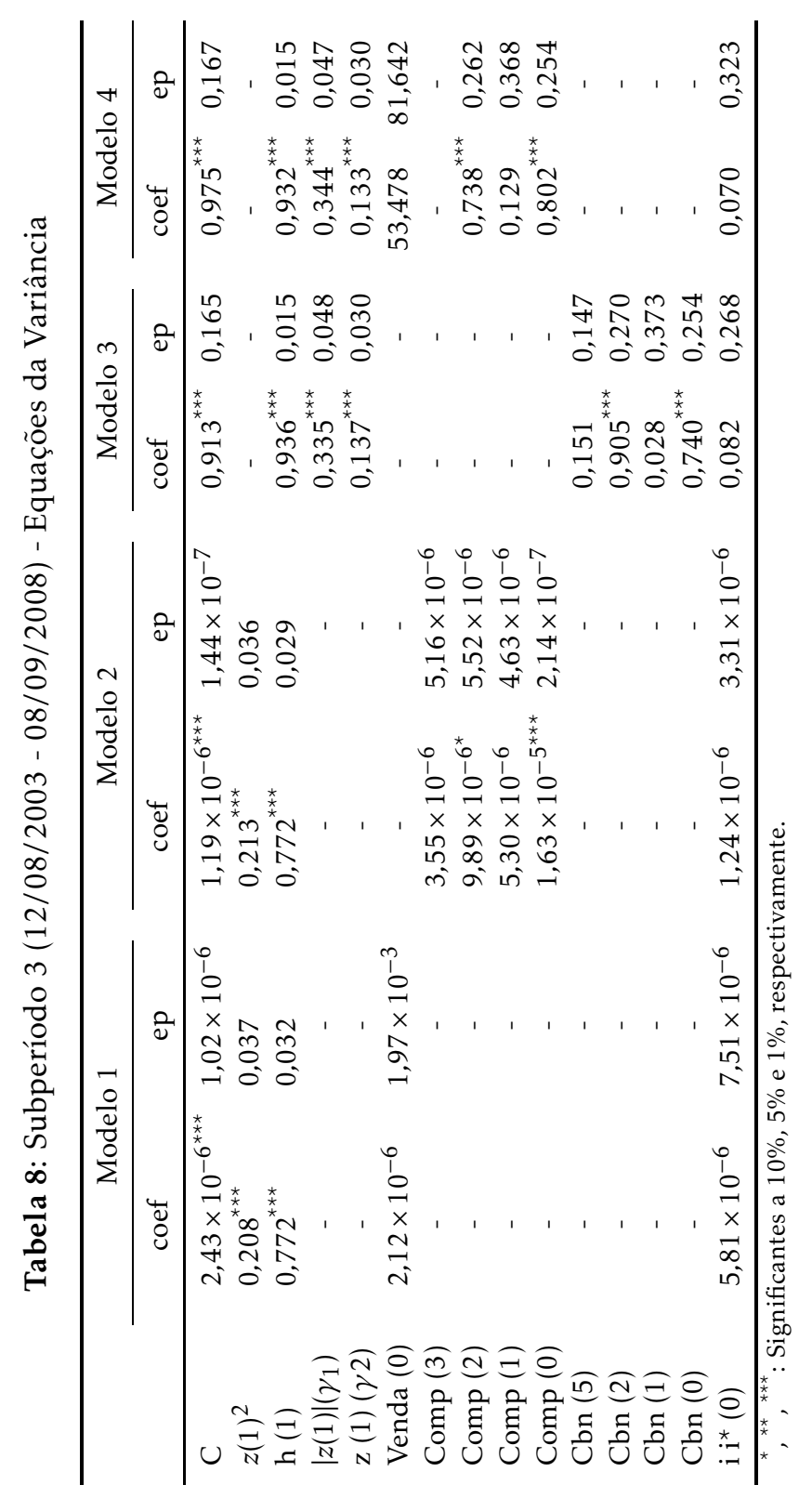




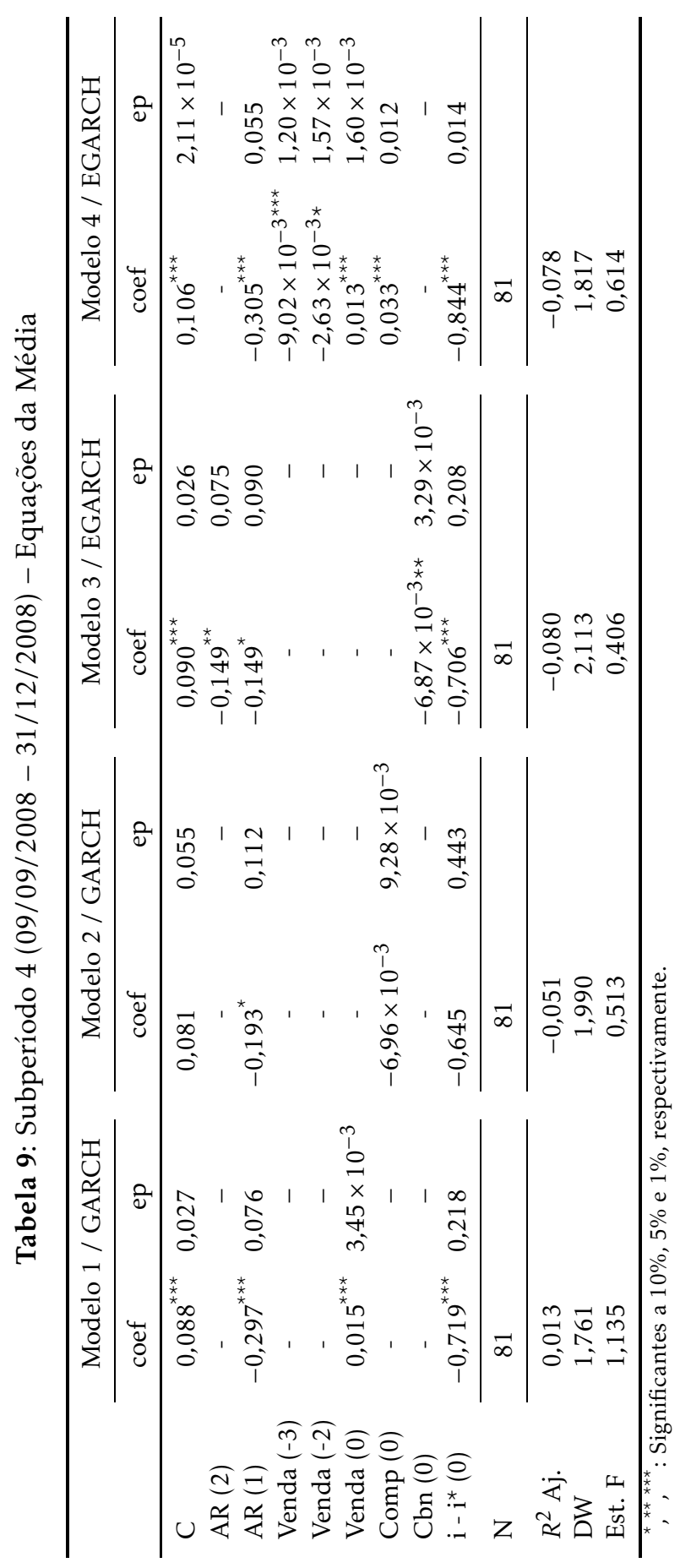




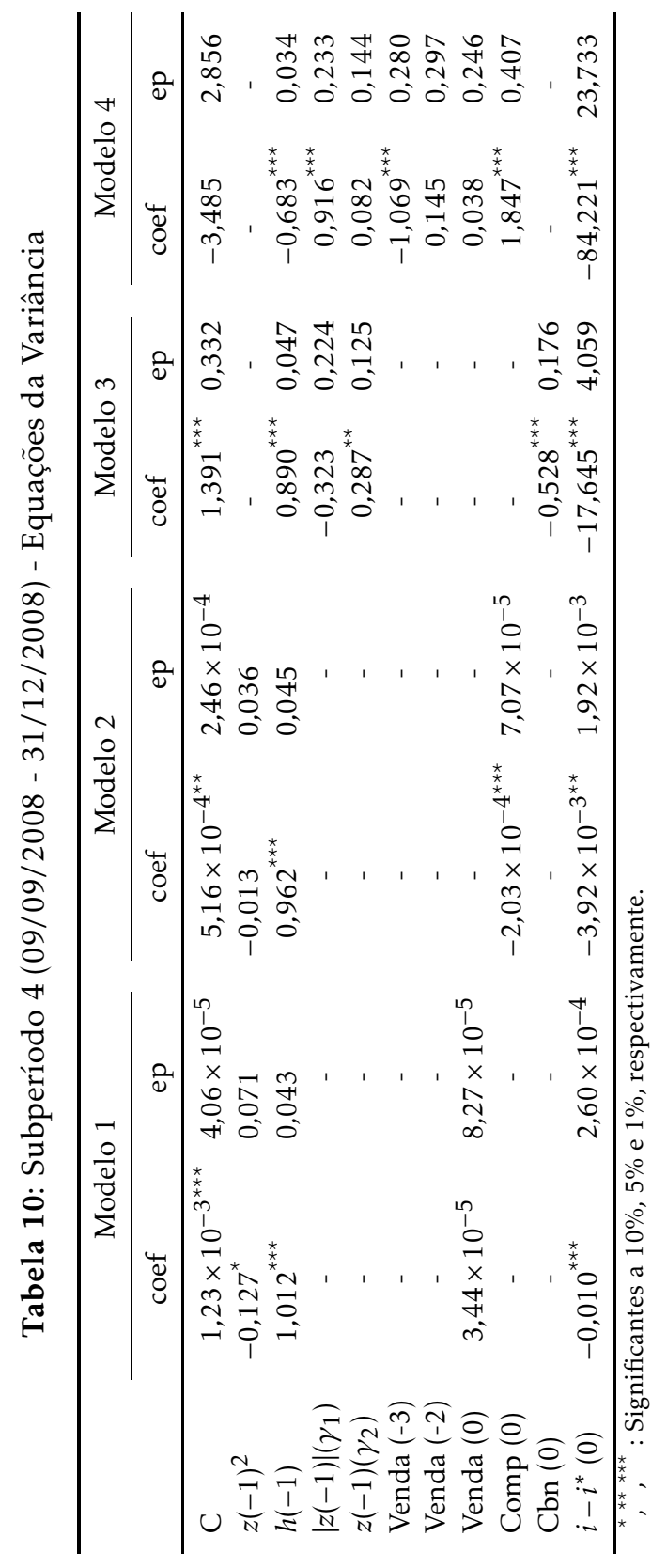


no dia seguinte. As intervenções nessa direção também parecem reduzir a volatilidade cambial.

Os modelos com intervenções combinadas identificam também um possível fenômeno de sobre-reação, dessa vez tanto em intervenções de venda como de compra. Isso é indicado pelo coeficiente negativo na data da intervenção e positivo na variável defasada em um dia. Também identificada assimetria nos choques: choques positivos têm maior efeito na variância condicional que choques negativos. Esse impacto é captado pelos coeficientes $\gamma 2$ positivos e significantes a $1 \%$ em ambos os modelos.

Os resultados obtidos indicam uma possível relação entre volatilidade e eficiência das intervenções cambiais: períodos de elevada volatilidade parecem relacionados com menor capacidade de as intervenções em afetarem o câmbio. Este resultado também suscita a hipótese de endogeneidade da intervenção: pode haver uma propensão a atuar quando a taxa de câmbio apresenta volatilidade elevada. A relevância dessa ótica é estudada por Kim et al. (2000) para o mercado de câmbio australiano, e por Araujo (2004), para o mercado brasileiro, ambos identificando possibilidade de endogeneidade na atuação dos bancos centrais.

O resultado da estimação por EGARCH mostra fortes indicativos de assimetria entre os choques, com tendência a choques positivos (que causam depreciação) apresentarem maior efeito na volatilidade que choques negativos. A intuição é de que as depreciações da taxa de câmbio ocorrem em movimentos mais rápidos que as apreciações, resultado similar ao encontrado em Meurer et al. (2007), também para o mercado brasileiro de câmbio.

Na subseção que segue, é feita uma comparação entre os resultados obtidos e o de outros estudos já realizados.

\subsection{Comparação com os resultados da literatura}

O presente trabalho indica a possibilidade de endogeneidade das intervenções cambiais, resultado também encontrado nos estudos empíricos de Kim et al. (2000) para o mercado australiano, e de Araujo (2004) para o mercado brasileiro. Não é possível definir a relação causal entre movimentos cambiais e intervenções, sendo que essa relação pode ocorrer tanto em uma forma ativa ( $\Delta$ intervenções $\rightarrow \Delta$ câmbio) como reativa ( $\Delta$ câmbio $\rightarrow \Delta$ intervenções). Nesse último caso, as intervenções ocorrem de acordo com uma função de reação (Kim 2003).

Os modelos EGARCH apontam a existência de assimetria entre os choques, com tendência a choques positivos (que causam depreciação) apresentarem maior efeito na volatilidade que choques negativos, resultado similar ao encontrado em Meurer et al. (2007), também para o mercado de câmbio brasileiro.

Foi encontrado também que o diferencial entre a taxa de juros interna e externa afeta consistentemente a taxa de câmbio, confirmando o papel da política monetária na determinação do câmbio e no próprio mecanismo de transmissão da política monetária, como proposto no estudo de Dominguez \& Frankel (1993). 


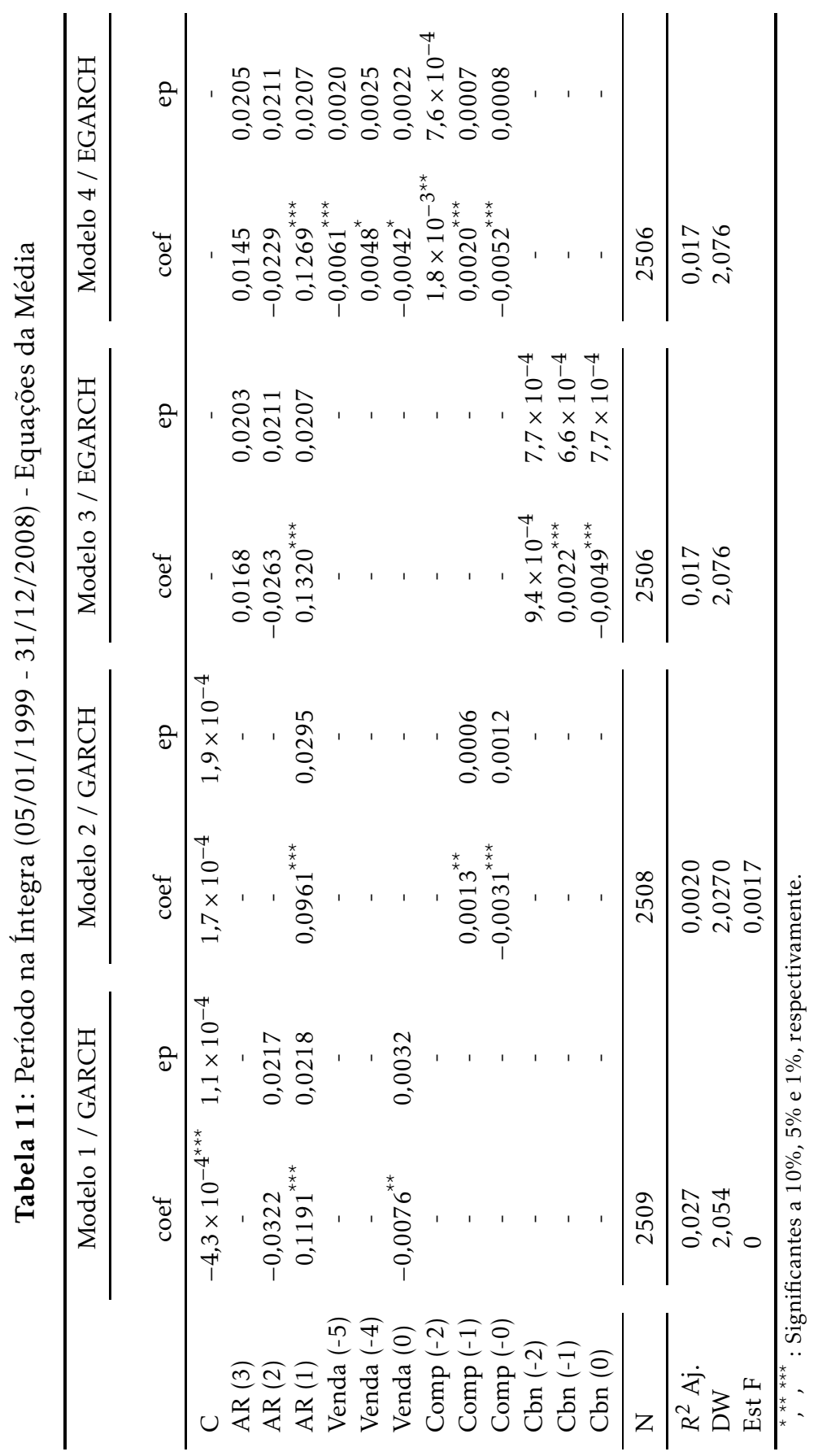




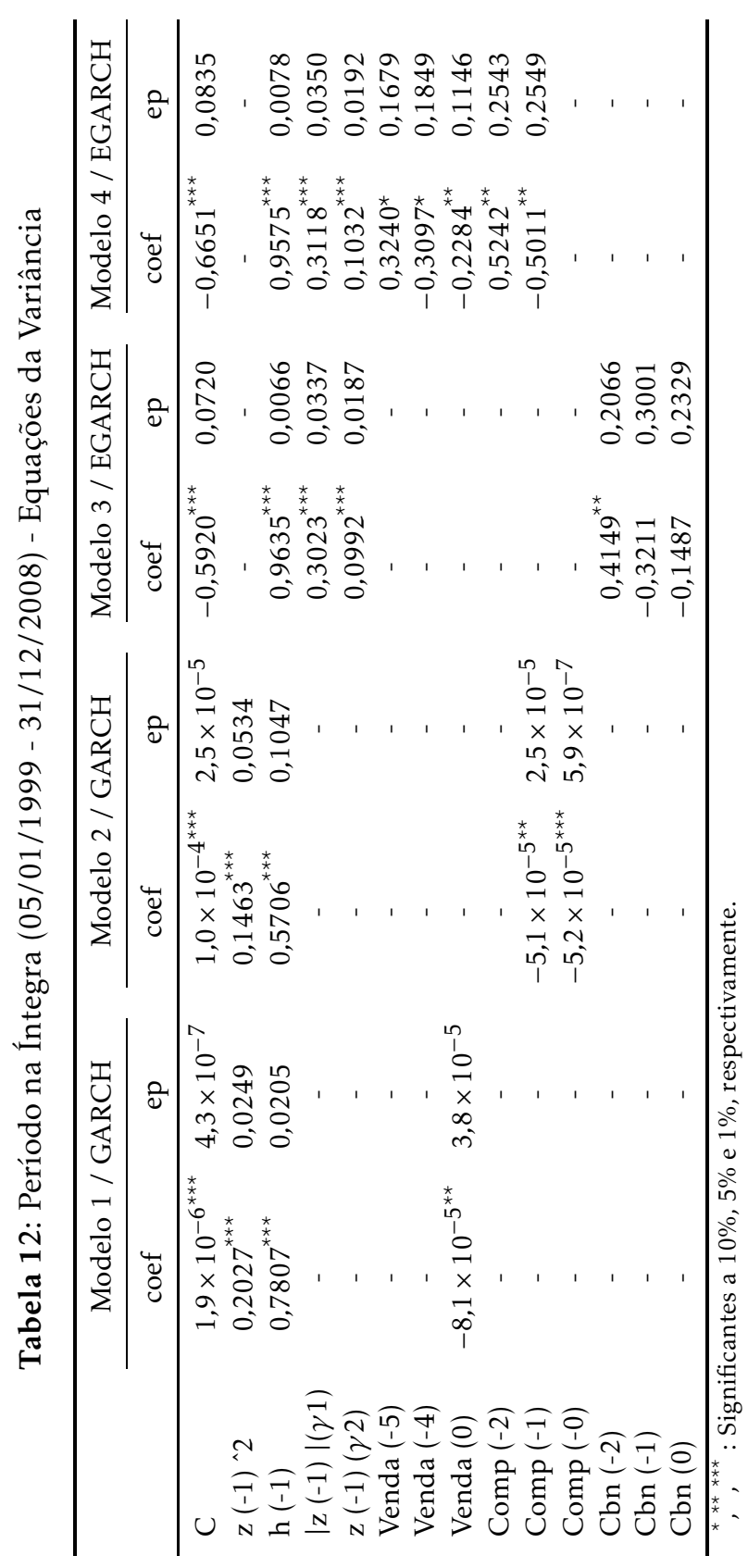




\section{Conclusões}

Este trabalho estudou as intervenções cambiais feitas por meio do mercado a vista, ocorridas no Brasil entre 1999 e 2008. Deve ser observado que, muitas vezes, as intervenções podem ocorrer sem necessariamente haver uma busca primária por efeitos na taxa de câmbio, já que a intenção por trás das intervenções cambiais em um regime de câmbio flutuante não é necessariamente explicitada.

Um fator comum obtido entre os diferentes subperíodos utilizados nas estimações é a existência de alguns resultados incongruentes entre a intervenção e o efeito no câmbio, o que é indício de endogeneidade da intervenção, que pode estar condicionada pelo comportamento do câmbio. Esta possibilidade está em linha com o discurso adotado pelas autoridades monetárias, de a intervenção ocorrer quando for oportuno, de forma a regularizar os mercados. Estudos empíricos como o de Kim et al. (2000) para o mercado australiano, e Araujo (2004), para o mercado brasileiro, também identificam a possibilidade de endogeneidade da intervenção.

Observa-se que nos períodos de maior instabilidade (subperíodos 2 e 4) a elevada volatilidade aliada à tendência da taxa de câmbio tornam menos eficientes as intervenções, caso o seu objetivo seja influenciar a cotação. Em períodos de volatilidade menos acentuada, como os intervalos 1 e 3 , as intervenções têm maior efeito sobre a taxa de câmbio.

De acordo com os modelos EGARCH, há fortes indicativos de assimetria entre os choques, com tendência a choques positivos (que causam depreciação) apresentarem maior efeito na volatilidade que choques negativos. A intuição é de que as depreciações da taxa de câmbio ocorrem em movimentos mais rápidos que as apreciações.

O comportamento do câmbio não é afetado apenas pela intervenção cambial ou esta reage somente ao comportamento da taxa. $O$ diferencial entre a taxa de juros interna e externa afeta consistentemente a taxa de câmbio, o que confirma o papel da política monetária na determinação do câmbio e no próprio mecanismo de transmissão da política monetária.

Uma extensão deste trabalho poderia ocorrer no sentido de explorar a existência de uma função de reação do Banco Central, baseada em subperíodos, de forma a analisar a dinâmica da função de acordo com as diferentes conjunturas econômicas e políticas.

\section{Referências Bibliográficas}

Alves, G. R. M. \& Simões, R. C. F. (2006), 'Implicações do novo regulamento de câmbio e capitais internacionais um ano após sua implantação', Revista de Negócios Internacionais 4, 25-30.

Araujo, J. D. P. (2004), Suavizando movimentos da taxa de câmbio ou adicionando volatilidade? um estudo empírico sobre intervenções do banco central no mercado de câmbio, Master's thesis, Pontifícia Universidade Católica do Rio de Janeiro.

Canuto, O. \& Holland, M. (2002), 'Flutuação cambial e metas inflacionárias em economias emergentes', Análise Econômica 20, 5-28. 
Dickey, D. \& Fuller, W. (1979), 'Distribution of the estimators for autoregressive time series with a unit root.', Journal of the American Statistical Association 74, 427-731.

Dods, J. L. \& Giles, D. E. A. (1995), 'Alternative strategies for 'augmenting' the dickey fuller test: Size-robustness in face of pre-testing', Journal of Statistical Computation and Simulation 53, 243-258.

Dominguez, K. (2006), 'When do central bank interventions influence intradaily and longer term exchange rate movements?', Journal of Internacional Money and Finance 25, 1051-1071.

Dominguez, K. \& Frankel, J. (1993), 'Does foreign exchange intervention matter? disentangling the portfolio and expectations effects for the mark', American Economic Review 83, 1359-1369.

Elliot, G., Rothenberg, T. J. \& Stock, J. H. (1996), 'Efficient tests for an autoregressive unit root', Econometrica 64, 813-836.

Fernandes, A. V. (2008), Microestrutura do mercado cambial brasileiro, Master's thesis, Pontifícia Universidade Católica do Rio de Janeiro.

Garcia, M. G. P. \& Didier, T. (2003), 'Taxa de juros, risco cambial e risco Brasil', Pesquisa e Planejamento Econômico 33, 253-297.

Gonçalves, C. E. S. \& Kankzuk, F. (2001), 'Prêmio de risco político', Estudos Econômicos 31, 429-457.

Guimarães, B. \& Gonçalves, C. E. S. (2007), Monetary policy, default risk and the exchange rate, Technical report, CEPR.

Humpage, O. F. (1984), Dollar intervention and the deutschemark-dollar exchange rate: A daily time-series model, Technical report, Federal Reserve Bank of Cleveland.

Kim, S. (2003), 'Monetary policy, foreign exchange intervention, and the exchange rate in unifying framework', Journal of International Economics 60, 355-386.

Kim, S., Kortian, T. \& Sheen, J. (2000), 'Central bank intervention and exchange rate volatility - australian evidence', Journal of International Financial Markets, Institutions and Money 10, 381-405.

Meurer, R., Moura, G. S. \& Nunes, M. S. (2007), 'O vencimento da dívida pública cambial influencia a taxa de câmbio? um estudo econométrico para o Brasil no périodo 2003-2004', Economia Aplicada 11, 55-72.

Meurer, R., Teixeira, F. W. \& Tomazzia, E. C. (2010), 'Eduardo c. efeitos das intervenções cambiais à vista na taxa de câmbio r/us de 1999 a 2008: um estudo de evento', Revista Brasileira de Finanças 8, 229-254.

Neely, C. J. (2005), An analysis of recent studies of the effect of foreign exchange intervention, Technical report, Federal Reserve Bank of St. Louis.

$\mathrm{Ng}$, S. \& Perron, P. (2001), 'Lag length selection and the construction of unit root tests with good size and power', Econometrica 69, 1519-1554. 
Obstfeld, M. (1990), The effectiveness of foreign-exchange intervention: Recent experience, 1985-1988", in W. Branson, J. Frenkel \& M. Goldstein, eds, 'International Policy Coordination and Exchange Rate Fluctuations', University of Chicago Press.

Reinhart, C. M. (2000), 'The mirage of floating exchange rates', American Economic Review 90, 65-70.

Sarno, L. \& Taylor, M. (2001), 'Official intervention in the foreign exchange market: Is it effective and, if so, how does it work?', Journal of Economic Literature $39,839-868$.

Schwartz, A. J. (2004), 'The rise and fall of foreign exchange market intervention as a policy tool', Journal of Financial Services Research 18, 319-339. 